\title{
Optical properties of mice skull bone in the 455- to 705-nm range
}

Haleh Soleimanzad

Hirac Gurden

Frédéric Pain 


\section{Optical properties of mice skull bone in the 455- to 705-nm range}

\author{
Haleh Soleimanzad, ${ }^{a}$ Hirac Gurden, ${ }^{\mathrm{b}}$ and \\ Frédéric Pain ${ }^{\mathrm{a}, *}$ \\ aUniversité Paris Sud, Université Paris Saclay, CNRS UMR8165, \\ Bat 440, Orsay F-91405, France \\ bUniversité Paris Diderot, CNRS UMR8251, Bat Buffon, \\ Paris F-75205, France
}

\begin{abstract}
Rodent brain is studied to understand the basics of brain function. The activity of cell populations and networks is commonly recorded in vivo with wide-field optical imaging techniques such as intrinsic optical imaging, fluorescence imaging, or laser speckle imaging. These techniques were recently adapted to unrestrained mice carrying transcranial windows. Furthermore, optogenetics studies would benefit from optical stimulation through the skull without implanting an optical fiber, especially for longitudinal studies. In this context, the knowledge of bone optical properties is requested to improve the quantitation of the depth and volume of imaged or stimulated tissues. Here, we provide experimental measurements of absorption and reduced scattering coefficients of freshly excised mice skull for wavelengths between 455 and $705 \mathrm{~nm}$. Absorption coefficients from 6 to 8 months mice skull samples range between $1.67 \pm 0.28 \mathrm{~mm}^{-1}$ at $455 \mathrm{~nm}$ and $0.47 \pm 0.07 \mathrm{~mm}^{-1}$ at $705 \mathrm{~nm}$, whereas reduced scattering coefficients were in the range of $2.79 \pm 0.26 \mathrm{~mm}^{-1}$ at $455 \mathrm{~nm}$ up to $2.29 \pm 0.12 \mathrm{~mm}^{-1}$ at $705 \mathrm{~nm}$. In comparison, measurements carried out on 4 to 5 weeks mice showed similar spectral profiles but smaller absorption and reduced scattering coefficients by a factor of about 2 and 1.5, respectively. () The Authors. Published by SPIE under a Creative Commons Attribution 3.0 Unported License. Distribution or reproduction of this work in whole or in part requires full attribution of the original publication, including its DOI. [DOI: 10.1117/1.JBO.22.1.010503]
\end{abstract}

Keywords: optical properties; skull; mice; absorption coefficient; reduced scattering coefficient; anisotropy coefficient.

Paper 160523LRR received Aug. 3, 2016; accepted for publication Jan. 4, 2017; published online Jan. 31, 2017; corrected Apr. 6, 2017.

\section{Introduction}

Several in vivo optical techniques used to study rat and mouse brain have been recently adapted to minimally invasive approaches in which imaging or stimulation is carried out through thinned bone skull without exposing the dura-matter or the brain tissues. These transcranial approaches allow repeated imaging of brain tissues over intervals ranging from seconds to months. ${ }^{1,2}$ In this context, the knowledge of skull bone's optical properties would allow (i) to better quantify the biophysical origins of the recorded optical signals and (ii) to quantify the volume and depths of tissues that are photostimulated. The skull is

*Address all correspondence to: Frédéric Pain, E-mail: pain @imnc.in2p3.fr a complex, translucent tissue. It is composed of different cell populations and structural tissue types. ${ }^{3}$ Several studies have been conducted on optical properties of human skull bone. The integrating sphere technique was used in the 520- to 960-nm spectral range. ${ }^{4}$ In another study, the bone optical properties were obtained at five wavelengths $(593,635,690,780$, and $830 \mathrm{~nm}) .^{5}$ A frequently cited study used spatially resolved diffuse reflectance at four wavelengths $(674,811,849$, and $956 \mathrm{~nm}){ }^{6}$ Pifferi et al. ${ }^{7}$ have measured the skull optical properties with time-resolved transmittance spectroscopy in the 650- to 1000 -nm window. Finally, Bashkatov et al. ${ }^{8}$ have studied optical properties of cranial bone in the infrared spectral range. In addition to these data on human skull, Firbank et al. ${ }^{9}$ have investigated pig skull in the 650- and 950-nm window. Surprisingly, data on the optical properties of skull bone of mice are scarce, whereas in vivo imaging of brain through the skull has become a major alternative to cranial window preparations, particularly for longitudinal studies. ${ }^{1,2}$ Here, we provide data on optical properties of fresh native skull bone of mice in the 455- to 705-nm range.

\section{Materials and Methods}

\subsection{Sample Preparations}

A first group of eleven C57B1/6 mice aged 6 to 8 months were used in this study. A second group of eight younger mice (mean age of 4 weeks) of the same strain was also studied. All experiments were performed in accordance with the European Directive 86/609/EEC regarding the care and use of laboratory animals. Immediately after mice sacrifice, their skulls were removed. The mean thickness of skulls for the first group was $0.34 \pm 0.05 \mathrm{~mm}$. For the second group, the mean thickness was $0.31 \pm 0.05 \mathrm{~mm}$. All samples were kept in a $0.9 \%$ saline solution and measured within $1 \mathrm{~h}$ after dissection. We have dealt with freshly resected, unprocessed bone, because polishing alters the surface state presumably increasing specular diffusion. Samples were characterized in two steps. First, measurements of reflectance and transmittance were carried out in the fresh, native state. Second, the same samples were immersed in pure $\mathrm{H}_{2} \mathrm{O}_{2}$ for $30 \mathrm{~min}$. Since haemoglobin is highly vulnerable to oxidative damage, $\mathrm{H}_{2} \mathrm{O}_{2}$ allows removing blood and having access to intrinsic bone optical properties. For all measurements, bone samples were placed between two microscope glass slides of 1-mm thickness, under minimal pressure in order to minimize rough surface effects and diminish specular reflection from the surface of the bone. Since the fresh samples are mechanically flexible, it was possible to flatten them between slides without breaking them.

\subsection{Spectrophotometric Measurements}

Spectroscopic measurements were carried out with a spectrophotometer (AvaSpec2048, Avantes, Netherlands) coupled to an integrating sphere (2" Integrating Sphere IS200-4, Thorlabs). The transmittance and reflectance were measured in the spectral range from 455 to $705 \mathrm{~nm}$. The entrance port of the integrating sphere is $12.7-\mathrm{mm}$ diameter. It is essential that the samples cover the entire port for accurate measurements. Consequently, we have reduced the entrance port diameter to $10 \mathrm{~mm}$ in diameter using a round washer with one face (interior) coated with a highly diffusive paint. The sample is illuminated with a tungsten-halogen light source (Thorlabs OSL1-EC) coupled to 
a $200-\mu \mathrm{m}$ diameter fiber collimated with a plug-in SMA collimator.

\subsection{Derivation of Absorption and Reduced Scattering Coefficients}

Absorption $\left(\mu_{\mathrm{a}}\right)$ and reduced scattering $\left(\mu_{\mathrm{s}}^{\prime}\right)$ coefficients of the samples were derived from measured values of the total transmittance and reflectance using the inverse double-algorithm (IAD). ${ }^{10}$ This well-established algorithm solves the radiative transport equation iteratively until the numerical fit and the experimental values of macroscopic reflection and transmission match together. ${ }^{10}$ The determination of the optical properties using IAD is a robust, well-validated technique. ${ }^{10,11}$ The two sets of data were measured before and after using $\mathrm{H}_{2} \mathrm{O}_{2}$. For each sample, experimental measurements at 26 wavelengths were used for absorption and reduced scattering coefficient calculation. In both groups, data were averaged over all mice for wavelengths between 455 and $705 \mathrm{~nm}$. We applied the Wilcoxon Signed Rank test using StatView (Version 5.0.1) to test the significance (statistical significance of $p<0.05$ ) of differences of the absorption and reduced scattering coefficient before and after using $\mathrm{H}_{2} \mathrm{O}_{2}$ for each wavelength.

\section{Results}

Images of representative mouse skull samples are shown in Fig. 1, before (a) and after immersion for $30 \mathrm{~min}$ in $\mathrm{H}_{2} \mathrm{O}_{2}$ (b). Before immersion in $\mathrm{H}_{2} \mathrm{O}_{2}$, blood is present in the bone tissues whereas after applying $\mathrm{H}_{2} \mathrm{O}_{2}$ the sample is clearer, almost bloodless. For the first group of mice, the average absorption coefficients before and after immersion in $\mathrm{H}_{2} \mathrm{O}_{2}$ are shown in Fig. 2. Coefficients varied from $1.67 \pm 0.28 \mathrm{~mm}^{-1}$ at $455 \mathrm{~nm}$ to $0.47 \pm 0.07 \mathrm{~mm}^{-1}$ at $705 \mathrm{~nm}$ before using $\mathrm{H}_{2} \mathrm{O}_{2}$. After $\mathrm{H}_{2} \mathrm{O}_{2}$ treatment, the absorption coefficients varied from $1.15 \pm 0.19 \mathrm{~mm}^{-1}$ at $455 \mathrm{~nm}$ to $0.53 \pm$ $0.07 \mathrm{~mm}^{-1}$ at $705 \mathrm{~nm}$. Data collected between 455 and $595 \mathrm{~nm}$ present a significant difference between both conditions $(n=11$, $P<0.05$ ). Data collected between 595 and $705 \mathrm{~nm}$ do not present any statistically significant differences $(n=11, P>0.06)$. This is likely due to a lower contribution of blood to absorption at higher wavelengths. The standard error of the mean shows moderate interindividual variability, which is independent of wavelength and is comparable to what was observed previously. ${ }^{12}$ As expected, the absorption coefficient for fresh skull presents blood absorption peaks characteristics of oxy-haemoglobin absorption in the visible range with two maxima, respectively, at 537 and $568 \mathrm{~nm}$. These two peaks strongly diminished after using $\mathrm{H}_{2} \mathrm{O}_{2}$. The average
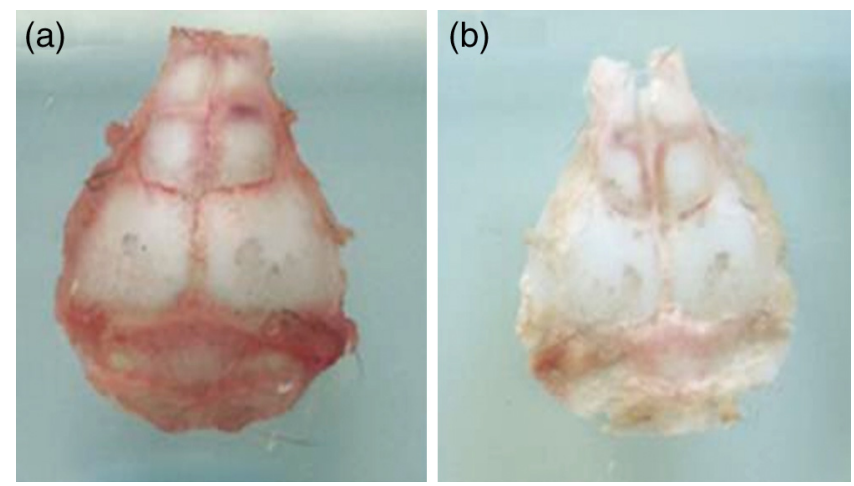

Fig. 1 The skull bone sample (a) before and (b) after treatment in $\mathrm{H}_{2} \mathrm{O}_{2}$ for 30 min.

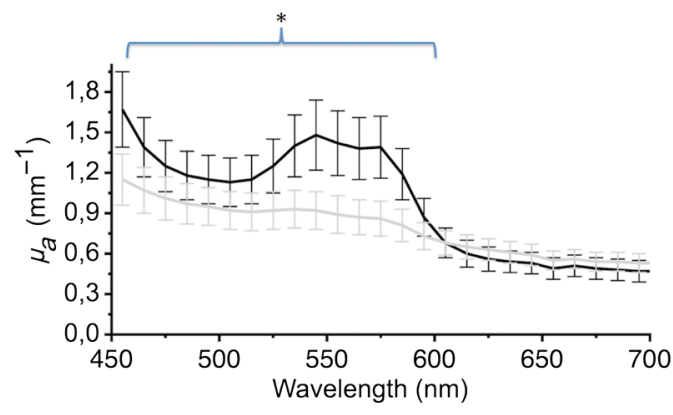

Fig. 2 Absorption coefficient of skull measured before (black) and after treatment with $\mathrm{H}_{2} \mathrm{O}_{2}$ (grey), error bars $\pm \operatorname{SEM}(n=11)$. The star indicates significant changes in the absorption coefficient $(p<0.05)$.

reduced scattering coefficients for the first group are shown in Fig. 3. Reduced scattering coefficients vary from $2.79 \pm$ $0.26 \mathrm{~mm}^{-1}$ at $455 \mathrm{~nm}$ to $2.29 \pm 0.12 \mathrm{~mm}^{-1}$ at $705 \mathrm{~nm}$ before $\mathrm{H}_{2} \mathrm{O}_{2}$ and varied from $4.17 \pm 0.34 \mathrm{~mm}^{-1}$ at $405 \mathrm{~nm}$ to $3.25 \pm 0.24 \mathrm{~mm}^{-1}$ at $705 \mathrm{~nm}$ after $\mathrm{H}_{2} \mathrm{O}_{2}$. Coefficients are significantly different before versus after $\mathrm{H}_{2} \mathrm{O}_{2}$ treatment for all the wavelengths $(n=11, P<0.05)$. The refractive index of hydroxyapatite (the main materials of the bone scatterers) is about 1.5 , whereas refractive index of interstitial fluid is 1.35 to $1.36 .^{13}$ Thus, the replacement of the interstitial fluid and bone by $\mathrm{H}_{2} \mathrm{O}_{2}$ should induce refractive index matching and, so, decrease of reduced scattering coefficient. A hypothesis that requires further investigation would be that $\mathrm{H}_{2} \mathrm{O}_{2}$ treatment leads to structural denaturation of bone, for example, by increasing bone porosity leading to the increase of the reduced scattering coefficient. A power law fit of the reduced scattering dependence with wavelength is classically observed for biological tissues. ${ }^{12,14}$ Here, it leads to $\mu_{\mathrm{s}}^{\prime}(\lambda)=32.529 \lambda^{-0.406}$ before $\mathrm{H}_{2} \mathrm{O}_{2}$ and $\mu_{\mathrm{s}}^{\prime}(\lambda)=116.05 \lambda^{-0.547}$ after $\mathrm{H}_{2} \mathrm{O}_{2}$ treatment ( $\mu_{\mathrm{s}}^{\prime}$ in $\mathrm{mm}^{-1}$ and $\lambda$ in $\mathrm{nm}$ ).

The comparison of the optical coefficients of the two groups of mice (young and old) is shown in Fig. 4. The optical coefficients showed similar dependency with the wavelengths for both groups. However, much lower absorption and reduced scattering coefficients are observed for younger mice for the entire range of wavelengths.

The knowledge of tissue autofluorescence (AF) is an important issue for wide-field fluorescence imaging (e.g., using calcium tracer) or in optogenetic experiments. ${ }^{15}$ An open question is the range of wavelengths to use to produce minimal bone AF. We have checked AF of the mouse skull (Fig. 5) with three excitation/emission filters sets (1) excitation: $470 \pm 40 \mathrm{~nm} / \mathrm{emission}$ $525 \pm 70 \mathrm{~nm}$, (2) Ex $545 \pm 25 \mathrm{~nm} / \mathrm{Em} 605 \pm 70 \mathrm{~nm}$, and (3) Ex $560 \pm 40 \mathrm{~nm} / \mathrm{Em} 630 \pm 75 \mathrm{~nm}$. The localization of

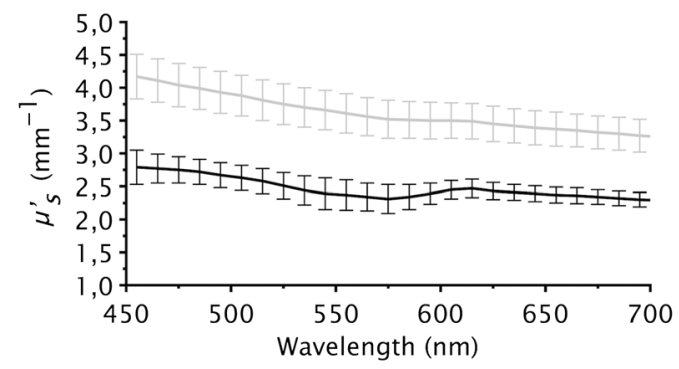

Fig. 3 Reduced scattering spectra of skull measured before (black) and after treatment with $\mathrm{H}_{2} \mathrm{O}_{2}$ (grey), error bars \pm SEM $(n=11)$. 

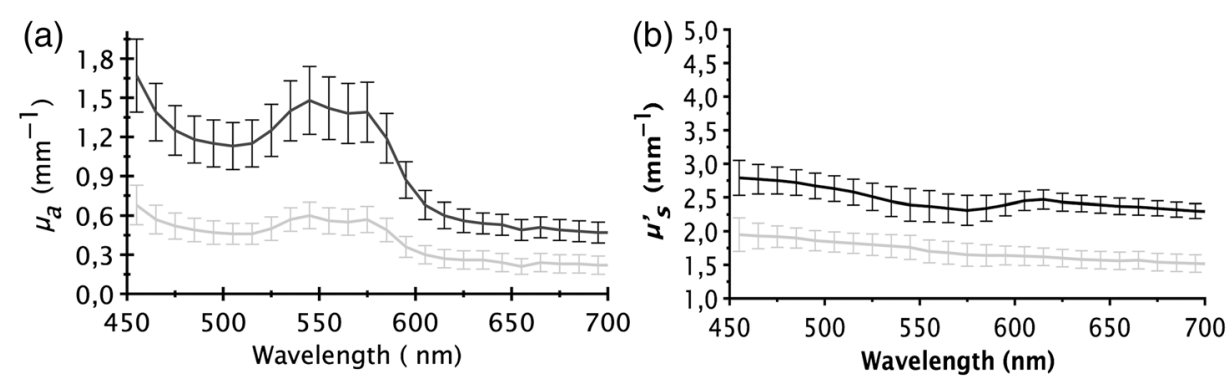

Fig. 4 Influence of age on the optical properties of the skull for 4-month-old mice ( $n=11$, black curve) versus 4-week-old mice ( $n=8$, grey curve). (a) Mean absorption coefficients \pm SEM. (b) Mean reduced scattering coefficients \pm SEM.

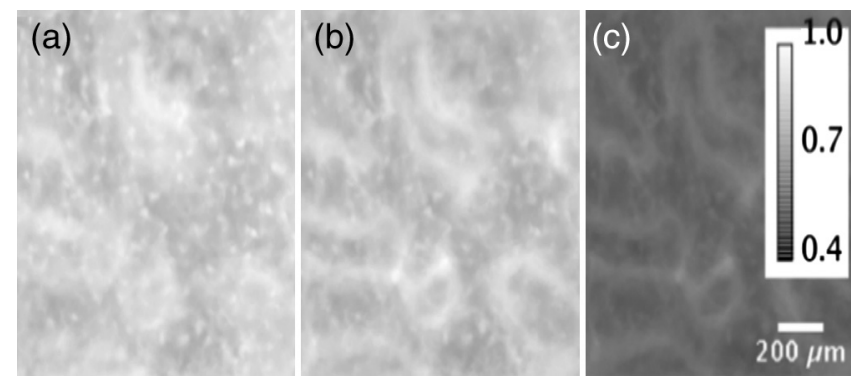

Fig. 5 Relative intensities of AF of bone skull for the three excitation/ emission filters sets: (a) blue excitation, (b) green excitation, and (c) red excitation (representative images from one mouse). Data have been normalized to the maximum intensity recorded in (a).

AF signals varied for the three filter sets indicating different physiological origins. Large structures appeared clearly under excitation with filter sets 2 and 3, whereas smaller structures are visible for filter set 1 . One can clearly identify large osteons structures of 200- to $300-\mu \mathrm{m}$ diameter, which are known as scatterers in bone tissues as well as nanostructures that cause the complex character of light scattering. The relative fluorescence intensity is similar for excitation under blue and green light, whereas intensity under red excitation is about $40 \%$ lower. These results suggest that brain-imaging techniques in the thinned skull preparation ${ }^{16,17}$ benefit from excitation and collection wavelength in the red/infrared region to minimize AF background noise. Regarding optogenetics, red-shifted opsins variants with spectral peaks near or above $600 \mathrm{~nm}$ are becoming available. $^{18}$

\section{Discussion and Conclusion}

In this study, the ex vivo optical properties of mouse skull bone were determined in the 455- to 705-nm window. To limit experimental variability, the same operator, using the same experimental set-up and standardized protocols, obtained all data. Regarding the variability of the optical properties of biological tissues, the literature shows relatively spread values for $\mu_{\mathrm{a}}$ and $\mu_{\mathrm{s}}^{\prime}$ for several tissues types mostly due to sample preparation and experimental method differences. ${ }^{14}$ Our findings can be considered in light of the results brought up previously. Similar reduced scattering coefficients were published by Firbank et al. $^{9}$ for the pig skull. However, our results show large differences in absorption coefficients measured at 455 to $705 \mathrm{~nm}$, with relatively strong discrepancy with the data at 650 to $950 \mathrm{~nm}^{9}$. Since the protocol used for pig skull bone is different from ours on mice, it is difficult to compare both datasets. $^{9}$ A source of potential errors in the IAD method is the assumptions made on the material homogeneity, thickness, and optical index. Estimations of the optical coefficients with IAD considering index from 1.36 to 1.55 and thicknesses from 0.29 to $0.39 \mathrm{~mm}$ led to changes in the estimations of $\mu_{\mathrm{a}}$ and $\mu_{\mathrm{s}}^{\prime}$ within interindividual variations. Data on the scattering of mouse skull are presented in a recent study on the feasibility of optical clearing for imaging through the intact skull. ${ }^{19,20}$ These data assume a constant absorption coefficient and anisotropy for all wavelengths to derive the scattering coefficients from a single collimated transmission measurement in the 500- to $900-\mathrm{nm}$ window.

Optical properties of skull change with age. This has two practical implications. First, young animals should be preferably chosen if imaging through the bone is considered. Second, longitudinal optical imaging studies through bone should take into account the change in optical properties if quantitative comparisons over time are required. Our data provide a solid basis for quantification improvement for imaging sessions carried out using the thinned bone preparation and could help further the development of skull optical clearing solutions. ${ }^{19}$ In addition, Monte Carlo simulations carried out in realistic three-dimensional geometries incorporating multiple tissues optical properties including skull would certainly help in (i) refining the knowledge of the depths and volume of the tissues that contribute to the biophysical imaging signals and (ii) designing experimental paradigms requiring noninvasive illumination of brain tissues through the skull, such as optogenetics or photostimulation.

\section{Disclosures}

No conflicts of interest, financial or otherwise, are declared by the authors.

\section{Acknowledgments}

Dr. Pain, Dr. Gurden, and Ms. H. Soleimanzad report grants from the French Ministry of Research and Education, during the conduct of the study.

\section{References}

1. G. Yang et al., "Thinned-skull cranial window technique for long-term imaging of the cortex in live mice," Nat. Protoc. 5(2), 201-208 (2010).

2. P. J. Drew et al., "Chronic optical access through a polished and reinforced thinned skull," Nat. Methods 7(12), 981-984 (2010).

3. G. Spatafora, "Optical mechanical and biochemical characterization of trabecular bone," PhD Thesis, University Politecnico di Milano (2014).

4. N. Ugryumova, S. J. Matcher, and D. P. Attenburrow "Measurement of bone mineral density via light scattering," Phys. Med. Biol. 49(3), 469483 (2004). 


\section{JBO Letters}

5. S. Tauber et al., "Lightdosimetric quantitative analysis of the human petrous bone: experimental study for laser irradiation of the cochlea," Lasers Surg. Med. 28(1), 18-26 (2001).

6. F. Bevilacqua et al., "In vivo local determination of tissue optical properties: application to human brain," Appl. Opt. 38(22), 4939-4950 (1999).

7. A. Pifferi et al., "Optical biopsy of bone tissue: a step toward the diagnosis of bone pathologies," J. Biomed. Opt. 9(3), 474 (2004).

8. A. N. Bashkatov et al., "Optical properties of human cranial bone in the spectral range from 800 to $2000 \mathrm{~nm}$," Proc. SPIE 6163, 616310 (2006).

9. M. Firbank et al., "Measurement of the optical properties of the skull in the wavelength range 650-950 nm," Phys. Med. Biol. 38(4), 503-510 (1993).

10. S. A. Prahl, M. J. C. van Gemert, and A. J. Welch, "Determining the optical properties of turbid media by using the adding-doubling method," App. Opt. 32(4) 559-568 (1993).

11. S. A. Prahl, "Light transport in tissues," PhD, University of Texas (1988).

12. M. Mesradi et al., "Experimental and analytical comparative study of optical coefficient of fresh and frozen rat tissues," J. Biomed. Opt. 18(11), 117010 (2013).
13. E. A. Genina, A. N. Bashkatov, and V. V. Tuchin, "Optical clearing of cranial bone," Adv. Opt. Tech. 2008, 1-8 (2008).

14. S. L. Jacques, "Optical properties of biological tissues: a review," Phys. Med. Biol. 58, R37-R61 (2013).

15. E. S. Boyden et al., "Millisecond-timescale, genetically targeted optical control of neural activity," Nat. Neurosci. 8(9), 1263-1268 (2005).

16. R. Vincis et al., "Dense representation of natural odorants in the mouse olfactory bulb," Nat. Neurosci. 15(4), 537-539 (2012).

17. V. Kalchenko et al., "Transcranial optical vascular imaging (TOVI) of cortical hemodynamics in mouse brain," Sci. Rep. 4, 5839 (2014).

18. J. Y. Lin et al., "ReaChR: a red-shifted variant of channelrhodopsin enables deep transcranial optogenetic excitation," Nat. Neurosci. 16(10), 1499-1508 (2013).

19. Y. Zhang et al., "Quantitative evaluation of SOCS-induced optical clearing efficiency of skull," Quantum Imaging Med. Surg. 5(1), 136-142 (2015).

20. J. Wang et al., "An innovative transparent cranial window based on skull optical clearing," Laser Phys. Lett. 9(6), 469-473 (2012). 